\title{
Experimental Verification of Super-resolution Optical Inspection for Semiconductor Defect by using Standing Wave Illumination Shift
}

\author{
S. Usuki ${ }^{1}$, H. Nishioka ${ }^{2}$, S. Takahashi ${ }^{2}$, and K. Takamasu ${ }^{2}$ \\ 1: Division of Global Research Leaders, Shizuoka University, Johoku 3-5-1, \\ Naka-ku, Hamamatsu, JAPAN \\ 2: Department of Precision Engineering, The University of Tokyo, Hongo 7-3-1, \\ Bunkyo-ku Tokyo, JAPAN \\ Phone: 81-53-478-1372 \\ Fax: 81-53-478-1372 \\ Email: dsusuki@ipc.shizuoka.ac.jp \\ URL: http://www.shizuoka.ac.jp/tenure/staffs/usuki.html \\ Name of conference: ISMTII2007 $\left(8^{\text {th }}\right.$ International Symposium on Measurement \\ Technology and Intelligent Instruments)
}

\begin{abstract}
:
Semiconductor design rules and process windows continue to shrink, so we face many challenges in developing new processes such as the less 100-nm design rule and the 300-mm wafer. The challenges have become more difficult, and the next generation of defect inspections is urgently needed. Optics and electron beams have been primarily used for the detection of critical defects, but both technologies have disadvantages. The optical inspection is generally not sensitive enough for defects at $100 \mathrm{~nm}$ or less, while the SEM inspection has low throughput because it takes a long time to scan $300 \mathrm{~mm}$. To find a solution to these problems, we have proposed a novel optical inspection method for the critical defects on the semiconductor wafer. First, we carried out theoretical examinations and computer simulations. As a result, the proposed method makes it possible to observe a structure with robustness and higher resolution beyond the Rayleigh limit. Second, we developed an apparatus for the basic experiment and carried out the super-resolution experiment using a line and space sample. As a result, the sample structures were clearly resolved beyond the Rayleigh limit and defects on the sample surface were detected with super-resolution.
\end{abstract}

Keywords: optical metrology and image processing, semiconductor inspection, standing wave illumination, image reconstruction, super-resolution

Abbreviations: SEM: Scanning Electron Microscopy, ITRS: International Technology roadmap for Semiconductors, CCD: Charge Coupled Device, PC: Personal Computer, NA: Numerical Aperture 


\section{Introduction}

According to the ITRS roadmap [1], a new generation of semiconductor defect inspection systems is urgently needed, and the requirement for sub-100-nm nodes and difficulty of defect-detection challenge are increasing exponentially with shrinking design rules [2]. One of the most important requirements is the defect detection of semiconductor wafer. Defects in the wafer inspection field include random defects like killer particles, clustered defects, and scratch defects. These defects deteriorate electrical chip performance and process yields in the factory line. In particular, inspection of patterned wafers is one of the key requirements for the next generation.

Optical methods and electron beams are conventionally used for semiconductor wafer inspection [3]. However, the inability of the optical inspection to handle the continuous miniaturization of interconnects has become a major issue. The electron beam is not useful for wider wafer inspection because it has low throughput properties. We focused attention on the optical inspection because it is nondestructive, and has high throughput and potential for high resolution for sub$100 \mathrm{~nm}$ inspection. In optical wafer inspection, resolution and defect detection beyond the Rayleigh limit are now required due to accelerating pattern miniaturization and the development of advanced semiconductor devices. One of the solutions to challenges in semiconductor optical inspection is the use of a shorter wavelength, which has been studied as a possible countermeasure against device miniaturization. However, the shortening of wavelength cannot keep up with the challenges and has a limit, so we propose the novel superresolution inspection technique. When a pattern is miniaturized and made dense, light reflected from the wafer becomes weak, and the captured image becomes dark with low contrast, so we must develop a high-resolution method capable of registering a large amount of optical information [4]. Our super-resolution inspection technique combines the standing wave illumination shift method with dark-field imaging technology to deliver optimal sensitivity for critical defect detection at 100-nm nodes and beyond without compromising on throughput. The standing wave illumination shift method enables the inspection optics to resolve patterns that the conventional method cannot. Nano-scale shift of standing wave illumination and obtaining multiple images with respect to each position of the 
standing wave illumination are keys to achieving resolution enhancement and higher sensitivity for defect detection.

\section{Methodology}

\subsection{Standing wave illumination shift and scattered light modulation}

The super-resolution obtained with the proposed method is based on multiple images detection with information regarding scattered light modulation [5]. We provide a schematic explanation of the standing wave illumination shift and the scattered light modulation in Fig. 1. The standing wave illumination is generated by two-beam interference with an objective lens. The standing wave illumination is scattered by the sample surface, and the scattered light is focused on the CCD imaging surface through the imaging lens. The standing wave is shifted at nanoscale based on the phase difference between the two beams in the illumination (Fig. 1(a)). Then, the scattered light is modulated by the shift of the standing wave illumination (Fig. 1(b)). The multiple images observed with respect to each shifted position of the standing wave illumination have frequency information and shift information of the standing wave illumination, so a super-resolution image is expected to be obtained with these multiple images.

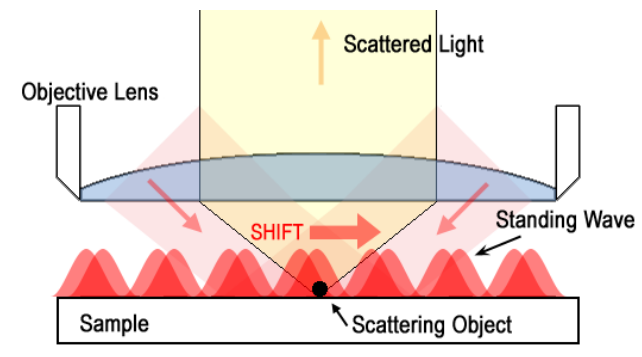

(a)

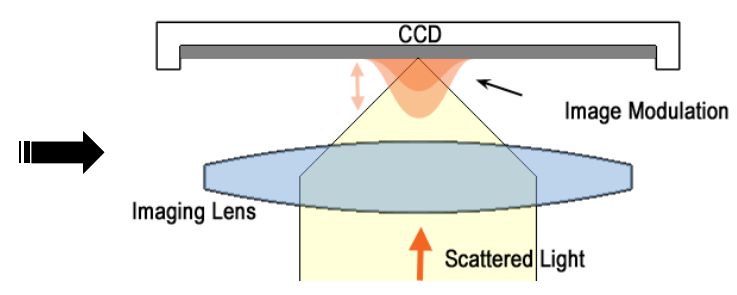

(b)

Fig. 1 Schematics of the standing wave illumination shift and the scattered light modulation

\subsection{Basic principle of super-resolution}

The practical explanation of the super-resolution obtained with proposed method is as follows. In the dark-field imaging, a scattering factor on the sample surface is considered as a point source of light. A conventional image on CCD is composed of image formation of the point sources of light. In the Fig. 2, the distribution of point sources resolved with super-resolution is calculated from image formations at the shifted positions of the standing wave illumination. 


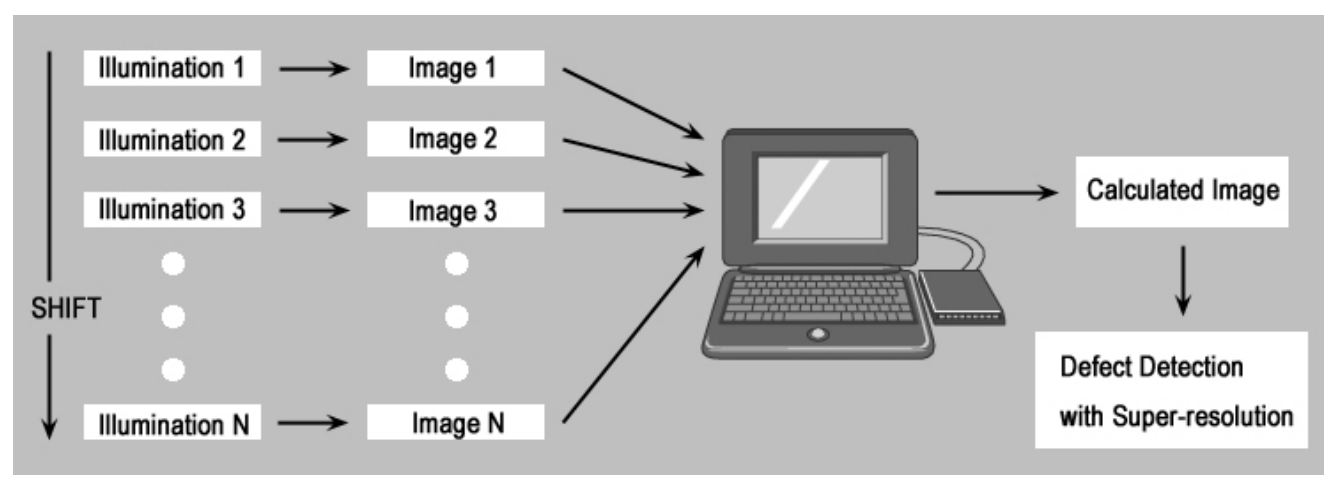

Fig. 2 Image (distribution) of point sources resolved with super-resolution is calculated from image formations 1 to $\mathrm{N}$ at the shifted positions of the standing wave illumination 1 to $\mathrm{N}$

The optical information of the illumination light is based on a nano-scale standing wave illumination shift, so we can expect superior resolution beyond the Rayleigh limit [6]. In this section, we build the model of physical phenomenon, and we propose a super-resolution method to reconstruct the scattering factor distribution from the amount of light detected with CCD.

In the proposed super-resolution method, we define the illumination intensity $I_{i}$ shown in Fig 3, and in this figure the number $i$ is the lateral position in the microscopic field. The scattering efficiency $\alpha_{i}$ shown in Fig. 4 is then defined.

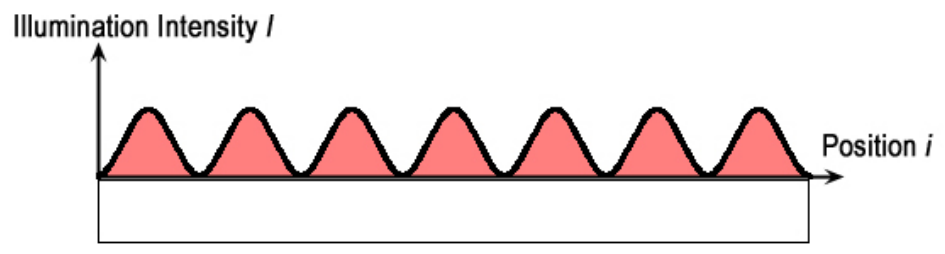

Fig. 3 Illumination intensity Ii; the number $\mathrm{i}$ is the lateral position in the microscopic field.

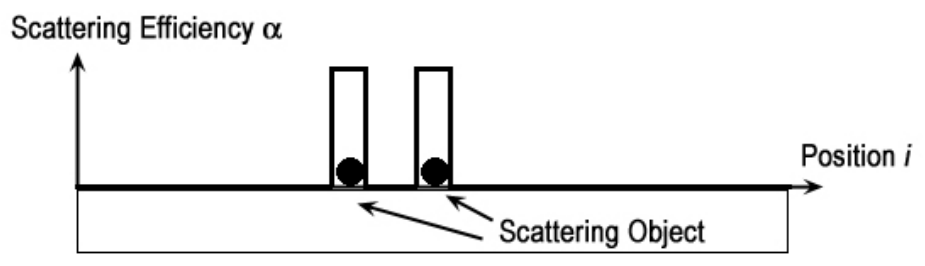

Fig. 4 Scattering efficiency is an optical value, and it is a transformational ratio from the illumination intensity to the scattering intensity.

The scattering efficiency is an optical value and is a transformational ratio from the illumination intensity to the scattering intensity. As we define the scattering 
intensity $S_{i}$ shown in Fig. 5, the relationship among the illumination intensity, the scattering efficiency, and the scattering intensity is given as

$$
S_{i}=\alpha_{i} \times I_{i}
$$

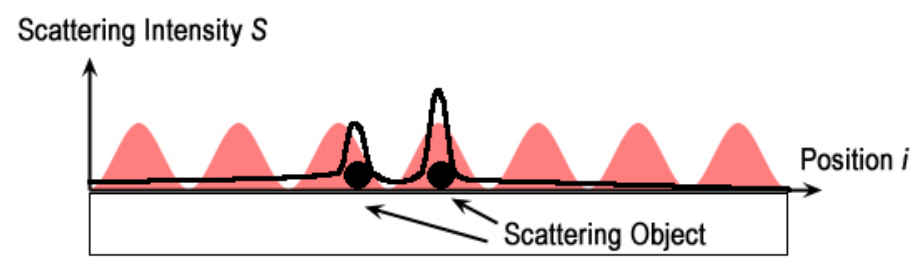

Fig. 5 Scattering intensity is the product value of the illumination intensity and the scattering efficiency.

In the imaging of the point source, it is necessary to consider the diffraction, so we introduce the diffraction contributing ratio $D$. This ratio is shown in Fig. 6, and the light detected with each pixel of the CCD camera is a part of the light amount of the airy disk. In the Fig. 6 , the scattered light for which the intensity is $S_{i}$, is detected with CCD pixels at position $j$ in the imaging field.

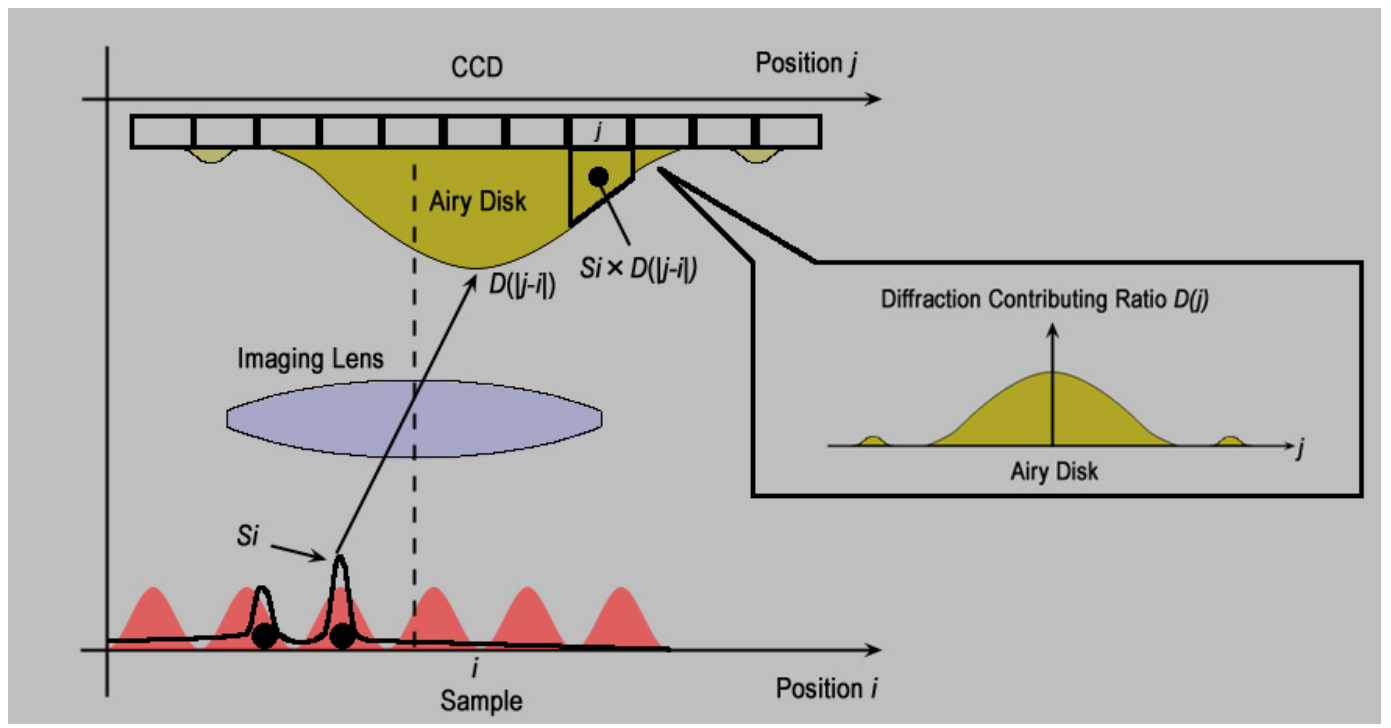

Fig. 6 Relation among the scattering intensity $S_{i}$, the diffraction contributing ratio $D(|j-I|)$ and the light amount $X_{j}$. The diffraction contributing ratio $D$ is given by the airy disk function.

The total light amount $\mathrm{Xj}$ detected with the CCD pixel is then given as

$$
X_{j}=\sum_{i} s_{i} \times D(|j-i|)=\sum_{i} \alpha_{i} \times I_{i} \times D(|j-i|)
$$


, in which $s_{i}$ is the scattering intensity at position $i$ and $D(|j-i|)$ is the diffraction contributing ratio at position $j$. The $X$ (the imaging light amount obtained by CCD), I (the illumination intensity determined by the generated standing wave), and $D$ (the diffraction contributing ratio determined by the optics) are known values, so $\alpha$ (the scattering efficiency) can be obtained by the equation (2) with the successive approximation. In this approximation, we set the initial value to a constant value, and calculate iterative operations until convergence. To obtain $\alpha_{i}$ established within a smaller area than the Rayleigh limit is to realize the superresolution.

\subsection{Super-resolution image reconstruction algorithm for post- processing}

In order to use spatial information from the standing wave illumination in carrying out the calculation, we constructed a super-resolution image reconstruction algorithm including iterative operations [7].

An optical imaging system is generally shift-invariant, so an image can be represented by convolution of a scattering object and a point spread function. The scattered light image $R(x)$ is determined from the sample distribution $a(x)$ and the illumination intensity $I(x)$ by the following equation.

$$
R(x)=P S F(x) \otimes(a(x) \cdot I(x))
$$

In this equation, $\operatorname{PSF}(x)$ is a point-spread function of the imaging optics, which represents the airy disk image by the diffraction and $\otimes$ is a convolution operator. The scattered light image $R(x)$ is modulated by shifting the standing wave illumination intensity $I(x)$ into different positions. The equation (3) is discretely described as follows.

$$
\begin{aligned}
& r_{i}=\sum_{j=1}^{N} p s f_{|i-j|} i_{j} a_{j} \\
& (1 \leq i \leq N)
\end{aligned}
$$

In this equation, $r_{\mathrm{i}}, p s f_{[\mathrm{i}-\mathrm{j} j}, i_{\mathrm{j}}$, and $a_{\mathrm{j}}$ are the image distribution, the point spread function, the illumination intensity distribution, and the objects distribution, respectively. Suffix $i$ represents a discrete position in the image plane and $j$ represents that in the object plane. The equation (4) can be described with using a matrix, as follows. 


$$
\begin{gathered}
\left(\begin{array}{c}
r_{1} \\
\vdots \\
r_{i} \\
\vdots \\
r_{N}
\end{array}\right)=\left(\begin{array}{ccccc}
p s f_{0} i_{1} & \cdots & p s f_{|1-j|} i_{j} & \cdots & p s f_{|1-N|} i_{N} \\
\vdots & \ddots & & & \vdots \\
p s f_{|i-1|} i_{1} & & p s f_{|i-j|} i_{j} & & p s f_{|i-N|} i_{N} \\
\vdots & & & \ddots & \vdots \\
p s f_{|N-1|} i_{1} & \cdots & p s f_{|N-j|} i_{j} & \cdots & p s f_{0} i_{N}
\end{array}\right)\left(\begin{array}{c}
a_{1} \\
\vdots \\
a_{j} \\
\vdots \\
a_{N}
\end{array}\right) \\
K=\left(\begin{array}{cccccc}
p s f_{0} i_{1} & \cdots & p s f_{|1-j|_{j}} i_{j} & \cdots & p s f_{|1-N|} i_{N} \\
\vdots & \ddots & & & \vdots \\
p s f_{|i-1|} i_{1} & & p s f_{|i-j|} i_{j} & & p s f_{|i-N|} i_{N} \\
\vdots & & & \ddots & \vdots \\
p s f_{|N-1|} i_{1} & \cdots & p s f_{|N-j|} i_{j} & \cdots & p s f_{0} i_{N}
\end{array}\right)
\end{gathered}
$$

In the equation $6, K$ is a coefficient matrix of imaging, so the imaging equation is given as

$$
R=K A
$$

, where $A$ is the object distribution matrix and $R$ the image matrix. The object distribution matrix $A$ is converted into image matrix $R$ with coefficient matrix $K$, so the imaging is represented by a linear simultaneous equation. The superresolution is realized by solving equation (7) for $A$, but a mathematical condition of the linear simultaneous equation of optical imaging is generally bad for calculation. Especially under actual conditions such as those with high levels of noise, convergence is difficult in the resolving calculation.

In order to solve this equation, we constructed an iterative super-resolution reconstruction algorithm in which one of the multiple images is occasionally used in the reconstruction and an assumed solution is then reconstructed. The block diagram of this algorithm is shown in Fig. 7 and the steps of the procedure are as follows.

(1) A sample is assumed to be a scattering factor distribution. The initial value of the assumed sample is a constant value $A_{\mathrm{i}}$. And the assumed sample is illuminated with the standing wave illumination which is computationally calculated. The initial calculated image matrix $R_{\mathrm{Si}}$ is given as

$$
R_{\mathrm{Si}}=K A_{\mathrm{i}}
$$


, where $A_{\mathrm{i}}$ is the assumed sample matrix and $K$ is the coefficient matrix shown in equation (7), and suffix $i$ is the image number.

(2) An actual image of the sample with standing wave illumination is observed by an experiment. The observed image $R_{\mathrm{Ei}}$ is the Rayleigh-limited image obtained by an imaging optics.

(3) The difference between the calculated and observed images is given as an error ratio with respect to each elements of image matrix. The error ratio $E_{\mathrm{i}}$ is shown in the following equation.

$$
E_{\mathrm{i}}(i)=\frac{R_{\mathrm{Ei}}(i)-R_{\mathrm{Si}}(i)}{R_{\mathrm{Si}}(i)}
$$

, where (i) represents an element of matrix (row number $i$ ).

(4) The error ratio $E_{\mathrm{i}}$ is fed back to the assumed sample. The assumed sample is reconstructed with the coefficient matrix $K$. A partition matrix to reconstruct the assumed sample $A_{\mathrm{i}}$ can be calculated with respect to each element of error ratio. The partition matrix $D_{\mathrm{i}}$ is given as the following equation.

$$
\begin{aligned}
& D_{\mathrm{i}}(i, j)=W(i, j) E_{\mathrm{i}}(i) \\
& W(i, j)=\frac{K(i, j)}{S(i)}, \quad S(i)=\sum_{j=1} K(i, j)
\end{aligned}
$$

In the equation (10) and (11), $W$ is the weighting coefficient matrix calculated from $K$ and $(i, j)$ represents an element of matrix (row number $i$ and column number $j$ ). The reconstructed sample $A_{\mathrm{i}+1}$ is then obtained as the following equation.

$$
A_{\mathrm{i}+1}=\left(I_{\text {ones }}+D_{i}\right) A_{\mathrm{i}}
$$

In the equation (12), $I_{\text {ones }}$ is a matrix of which all elements are one.

(5) The initial assumed sample $A_{\mathrm{i}}$ is replaced by the reconstructed sample $A_{\mathrm{i}+1}$. The reconstructions are applied to the other shifted position of the standing wave illumination. The reconstruction times are $N$ if the number of images is $N$. 
(6) The reconstruction (1)-(5) is iteratively applied to decrease the error ratio and to converge the solution.

The image expected from the assumed sample is compared with the actual image, as obtained experimentally, to obtain the next assumed sample with the weight of the coefficient matrix. By using the reconstruction method described above, the assumed sample approaches the object distribution of the actual sample to obtain a super-resolution image. We expect to achieve super-resolution, because the nanoshifts of standing wave illumination that are modulated in approximately halfwavelength scale include high-frequency spatial information which causes a change in the scattered light images.

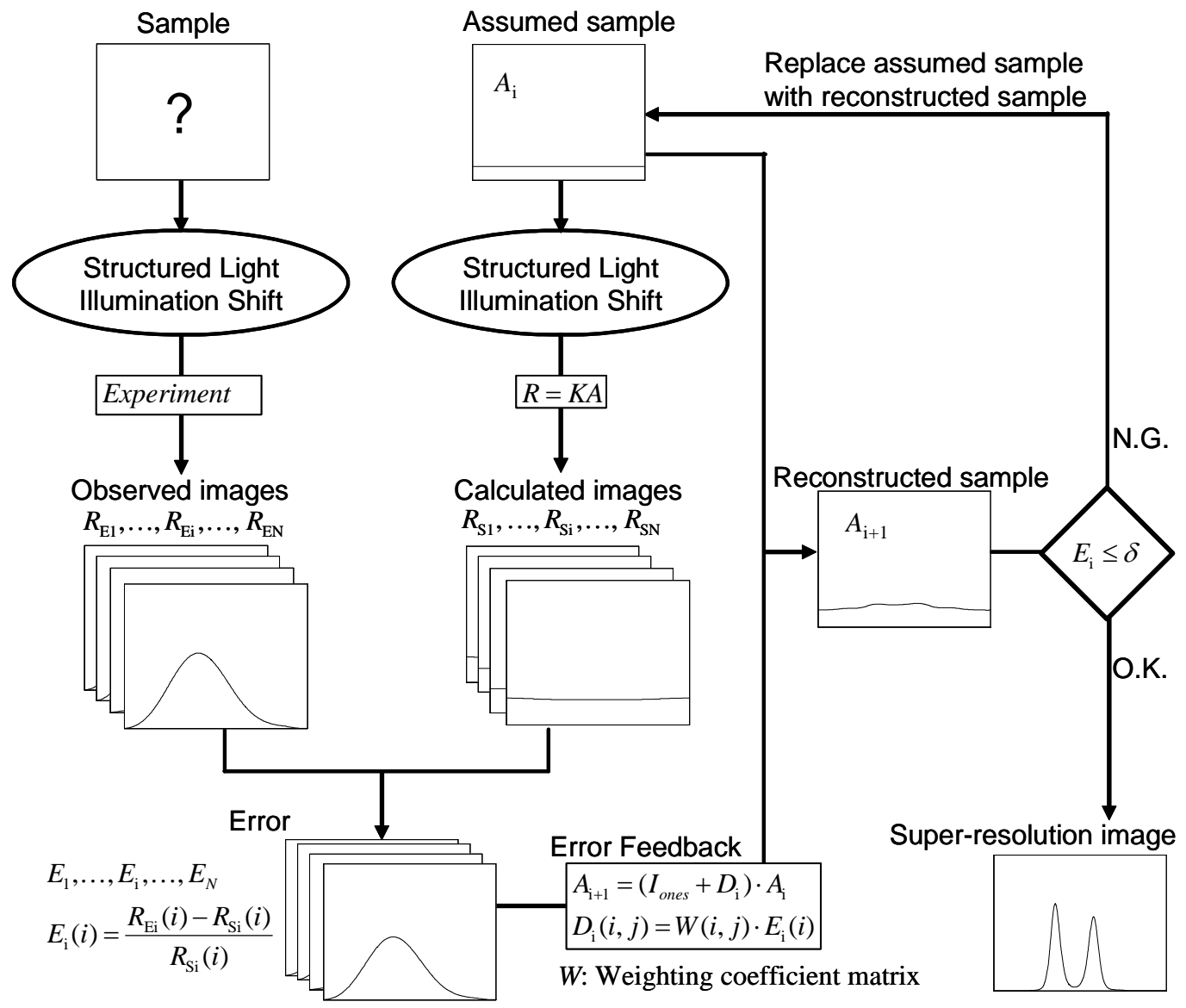

Fig. 7 Block diagram of iterative super-resolution reconstruction based on multiple shift of illumination 


\section{Computer simulation}

\subsection{Verification of resolution properties of the super-resolution algorithm}

The super-resolution algorithm was numerically applied to the super-resolution imaging. A two-point scattering object was used as a sample in the verification. A simulation model is shown in Fig. 8, and the conditions of the simulation are shown in Table 1. The sampling size in the simulation was small enough to avoid a discretization error. The numerical aperture of the objective was 0.95, and the wavelength of the light source was $488 \mathrm{~nm}$, so the Rayleigh limit of the imaging optics was $313 \mathrm{~nm}$ in the simulation. Eight shift times were used in the standing wave illumination to verify the algorithm in which the multiple shifts were applied. The iteration times of the reconstruction was set to be 10000 to converge the solution. The multiple images and the super-resolution image are shown in Fig. 9, in which the gap of the 2-point scattering objects is $313 \mathrm{~nm}$ (the Rayleigh limit of imaging optics). In the Fig. 9, the correspondence of the image modulation to the illumination distribution was confirmed, and the separation of the 2-points was clearly identified. Based on the results shown in Fig. 9, we suggested the capability for resolving the two-point scattering objects, which is not resolved with a conventional optical microscopy. The super-resolution was therefore numerically realized by the constructed algorithm.

Then, in order to confirm the possibility for resolution beyond the Rayleigh limit, the gaps of the 2-point scattering object were set to be $250 \mathrm{~nm}$ (the Sparrow limit of the imaging optics) and $169 \mathrm{~nm}$ (the resolution limit assumed from the spectrum of the standing wave illumination [7]). The resulting super-resolution images are shown in Fig. 10. In the case of 250-nm gapped 2-point, the flat part was confirmed around the center of the conventional image distribution, so it was corresponding to the Sparrow limit of resolution. The differentiation of 2-points from 1-point was difficult in the case of 169-nm gapped 2-point. On the other hand, in both cases, the 2-points were able to be independently identified in the super-resolution images. 


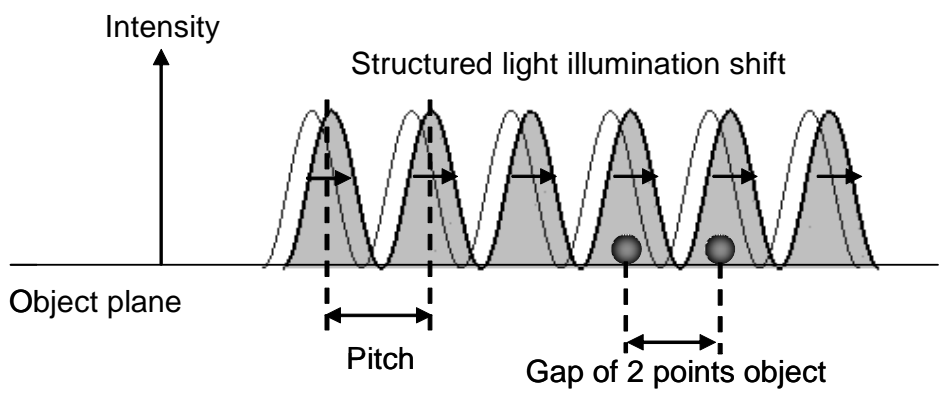

Fig. 8 Simulation model for resolving 2-point object

Table 1 Parameter setup for super-resolution

\begin{tabular}{c|c}
\hline \hline Wavelength of source & $488 \mathrm{~nm}$ \\
Pitch of structured light illumination & $300 \mathrm{~nm}$ \\
Sampling size & $1.2 \mathrm{~nm}$ \\
NA of objective & 0.95 \\
Rayleigh limit & $313 \mathrm{~nm}$ \\
Shift times & 8 \\
Shift step size & $30 \mathrm{~nm}$ \\
Iteration Times & 10000 \\
Gap of 2-point object & $313 \mathrm{~nm}, 250 \mathrm{~nm}, 169 \mathrm{~nm}$ \\
\hline
\end{tabular}



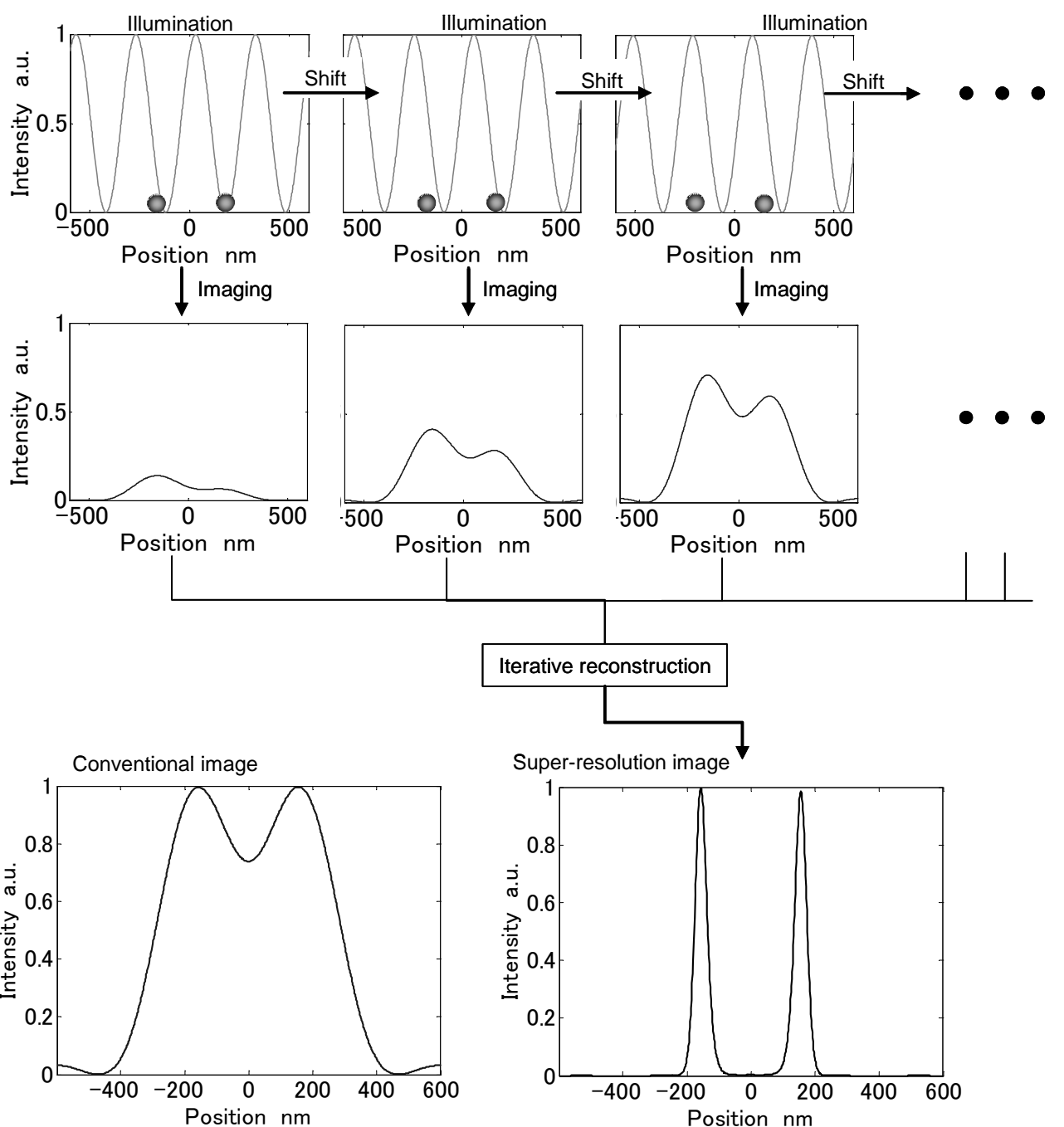

Fig. 9 Resolving the Rayleigh limited gap (313nm) by structured light illumination shift and iterative reconstruction of multiple images 

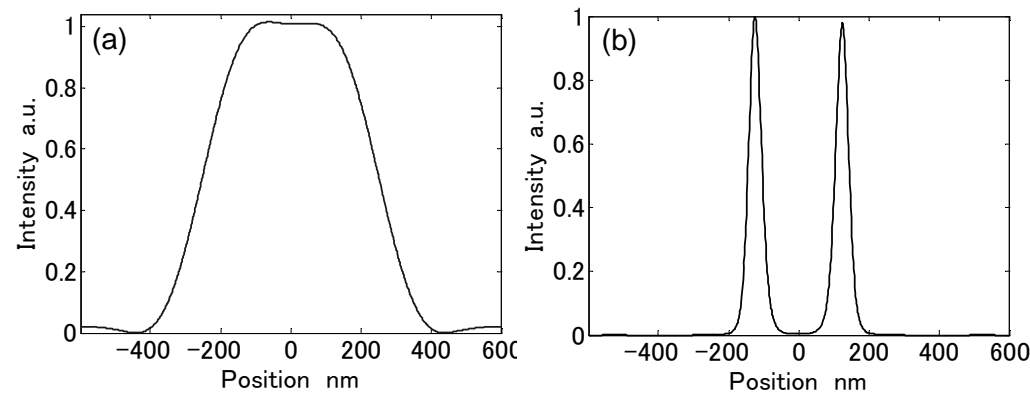

(A) Result obtained from $250 \mathrm{~nm}$ gapped 2 points object
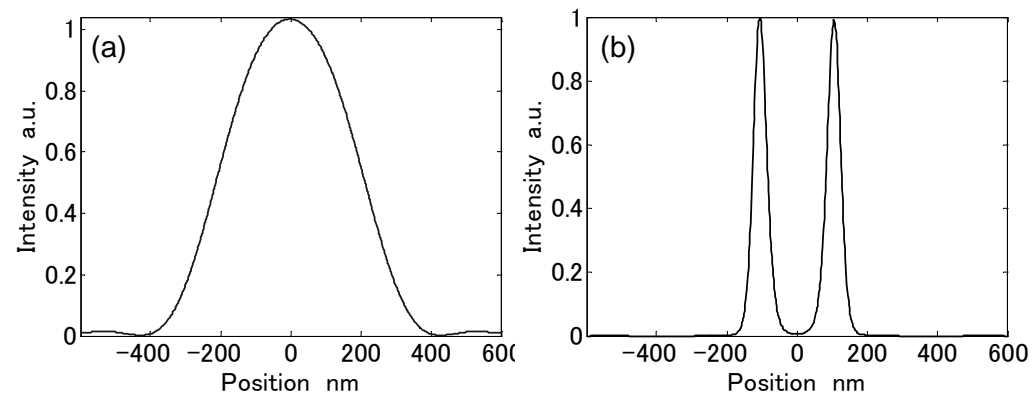

(B) Result obtained from169nm gapped 2 points object

Figure 10 Comparison of conventional image and super-resolution image; (a) Conventional image of 2-point object, (b) Super-resolution image of 2-point object

\subsection{Improvement of noise-robustness using multiple images}

Noise-robustness is one of the most important factors in applying the proposed method to semiconductor inspection. In this section, random noise out of various kinds of noise was taken into special consideration, because systematic noises can be reduced with a calibration. And other conditions of the simulation are the same ad those shown in Table 1.

The relationship between noise-robustness and illumination shift times (number of images) was investigated by numerical simulation. The conditions of the simulation are the same as those described above, and 30\% random noise was added to all images observed in the case of a 169 nm-gapped 2-point scattering object. An observed image of 2-point scattering objects with 30\% random noise is shown in Fig. 11. In this simulation, the shift step size was set to be a constant 30 $\mathrm{nm}$ to clarify the effects of the shift of the standing wave illumination.

The super-resolution images with various shift times are shown in Fig. 12. In the case of shift time 0 , the super-resolution image distribution was irregular by random noise mixed in observed images, but the distribution was improved to a clearly resolved distribution by increasing the shift times to 20 . 
Generally, according to the research by Frohn et al [6], two shifts of the standing wave illumination (3 images) are sufficient to realize the super-resolution, but an additional increasing shift of illumination contributes to improvement of resolution in cases of noise. In order to quantitatively evaluate the effects of increasing the shift times, simulations with various shift times were implemented, in which the peak-to-valley ratio of the super-resolution image distribution $(V / P)$ was a barometer of the noise-robustness. The means of calculated V/P with standard deviation are plotted against shift times in Fig. 13. The noise-robustness was improved with redundant shift of the standing wave illumination and it was confirmed that $169 \mathrm{~nm}$-gapped 2-point objects could be clearly resolved in 30\% random noise by using more than 10 shifts. These results indicate that the phase condition was completed because the number of shifts was 10 when the pitch of the standing wave illumination was $300 \mathrm{~nm}$ and the shift step size was $30 \mathrm{~nm}$.
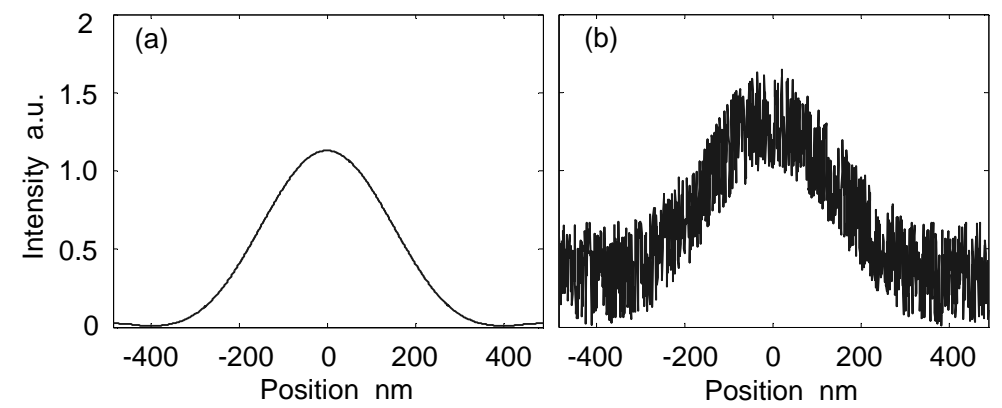

Fig. 11 Obtained image for super-resolution; (a): Noiseless image, (b): Image with 30\% random noise

(a) Shift times $=0$

(b) Shift times $=6$

(c) Shift times $=20$

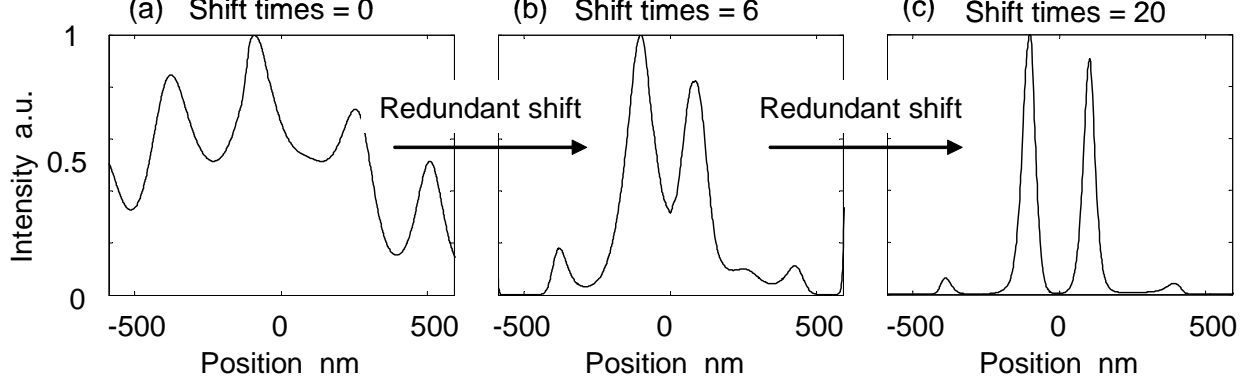

Fig. 12 2-point super-resolution images under noise; Shift times: 0(a), 6(b), 20(c) 


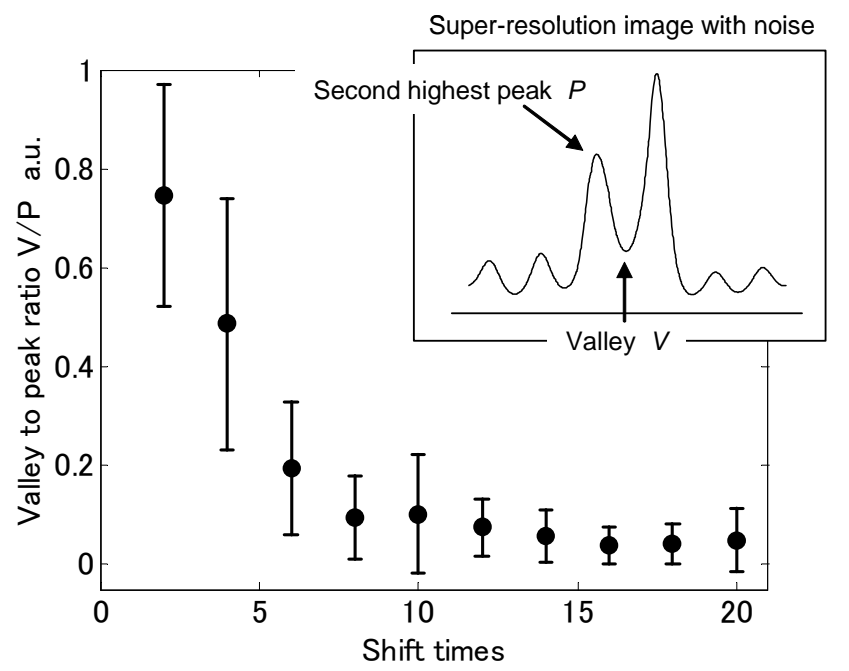

Fig. 13 Enhancement of robustness by redundant shift

\section{Experimental verification}

\subsection{Experimental apparatus}

In order to experimentally verify the super-resolution method, we developed the experimental apparatus for the standing wave illumination, the nano-shift of the standing wave, and the dark-field imaging of the scattered light [8]. A schematic diagram of the system of the super-resolution optical inspection using standing wave illumination shift is shown in Fig. 14. The PC in the figure controls the CCD, the PZT, and the XYZ scanner, and it is used for super-resolution postprocessing. In the Fig. 14, the beam splitter 1 divides a beam of light into two orthogonal beams. The two beams are collimated and face the microscopic field of the high power objective lens with a high numerical aperture thorough mirror 1 , mirror 2, mirror 3, mirror 4, lens 1 , lens 2 , lens 3, lens 4 , and beam splitter 2 . The two collimated and facing beams interfere on the sample surface and generate standing wave illumination.

The scattered light from the sample surface with the standing wave illumination is focused on the CCD imaging surface through the objective lens, lens 5, beam splitter 2, filter and tube lens. In the imaging optics, the objective lens and the lens 5 are set for the infinity correction optics, and the tube lens is set for the finite optics. 
In the Fourier transform plane of the lens 5, the filter rejects the transmitted light that is the regular reflection of the illumination light, so we can obtain only light from the scattering factor on the sample surface with high contrast. This method is like dark-field imaging and laser scattering microscopy, so it has an optically high signal-noise ratio.

The standing wave illumination produced by the illumination optics is shifted at nano-scale with the piezo-electric actuator attached to mirror 1 . The light axial position of the mirror 1 is displaced at nano-scale with the piezo-electric actuator, which produces phase the difference between the two-beams in the illumination optics.

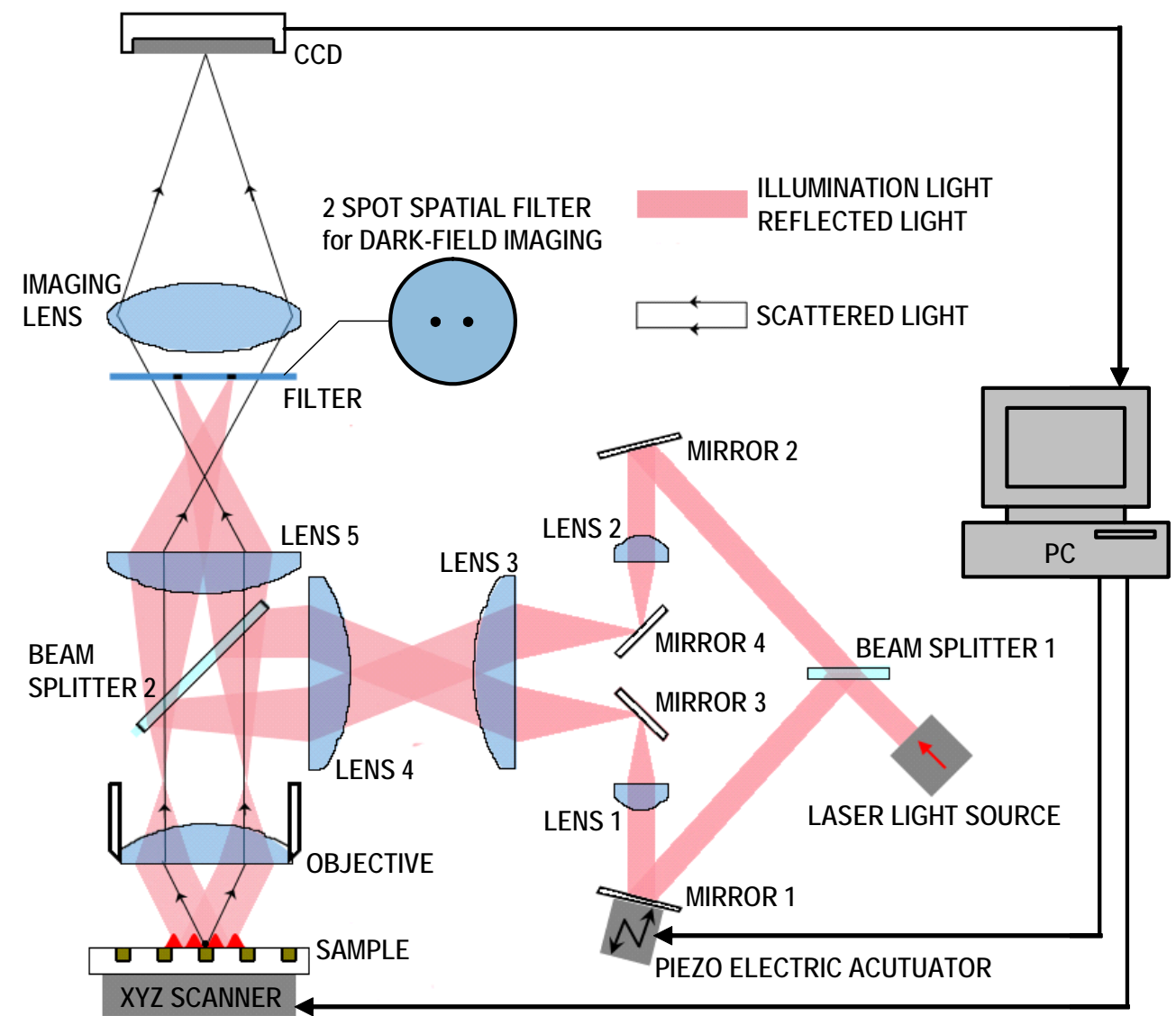

Fig. 14 Schematic diagram of the optical system of the super-resolution optical inspection for semiconductor defects using a standing wave shift. These optics include the standing wave illumination generator, the standing wave shift system and the dark-field imaging system using the filter for transmitted light rejection. 


\subsection{Confirmation of standing wave illumination and scattered light modulated by standing wave illumination shift}

For fundamental verification of the super-resolution method, we performed the basic experiment using the developed apparatus in the former section. The experimental setup is shown in Table 2. It is expected that the super-resolution method will make it possible to observe a structure with higher resolution than conventional resolution, depending on the NA of the objective lens. And for verification of the essence of the method, we use an objective lens with relatively low NA (0.46). The super-resolution method assumes the standing wave illumination and the scattered light modulation by the nano-shift of the illumination. The discrete sample is suitable for the scattered light modulation and examination of resolution, so we use a standard particle in the experiment. The sample with a mirror-plane pattern on the wafer is shown in Fig. 15(a). The sample image is observed with an optical microscope for which the magnification is 20. The sample was illuminated with the illumination optics of the developed apparatus. In Figs. 15(b) and 15(c), an image with uniform illumination and an image detected with standing wave illumination are shown, respectively. We confirmed the pitch (peak-to-peak intensity) of the standing wave illumination, which is approximately $1200 \mathrm{~nm}$. The pitch approximately conformed to the theoretical pitch of the standing wave calculated with the facing angle of the two beams. The 3D magnified view of the standing wave illumination is shown in Fig. 15(d). It was found that the standing wave illumination had an intensity distribution like a sine wave.

In the Fig. 16, we plot the detected light intensity of the peak position of the particle image against the standing wave shift times, in which the shift time is corresponds to an approximately 60-nm lateral shift of the standing wave illumination. We confirmed the linearity of the scattered light intensity against the scattering factor of the particle because the modulation of the scattered light intensity was related to periodic distribution of the standing wave illumination. In addition, we found that the pitch of the standing wave was approximately 1200 $\mathrm{nm}$ because the scattered light intensity was approximately 4.75 times alternating between bright and dark when the total shift was approximately $6 \mu \mathrm{m}$. 
Table 2 Experimental setup of the optical system

\begin{tabular}{c|c}
\hline \hline Wave length of source: $\lambda$ & $488 \mathrm{~nm}$ \\
Positioning accuracy of PZT: $p_{\text {PZT }}$ & $1 \mathrm{~nm}$ \\
Numerical aperture of objective: $N A$ & 0.46 \\
Spot size of Spatial filter for dark field imaging: $s_{\text {dark }}$ & $0.5 \mathrm{~mm}$ \\
Pixel size of cooled CCD camera: $s_{\mathrm{CCD}}$ & $8.3 \mu \mathrm{m}$ \\
\hline \hline
\end{tabular}

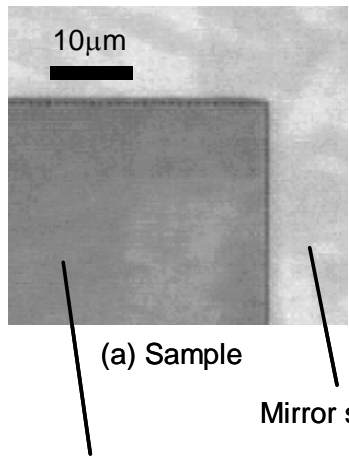

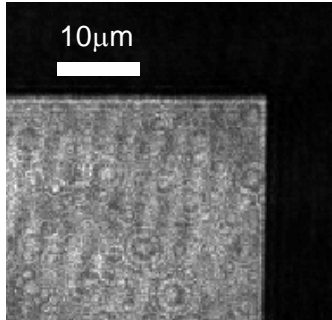

(b) Uniform illumination

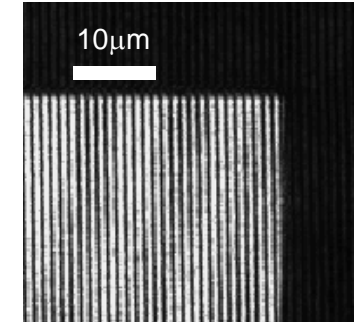

(c) Standing wave illumination

Scattering surface

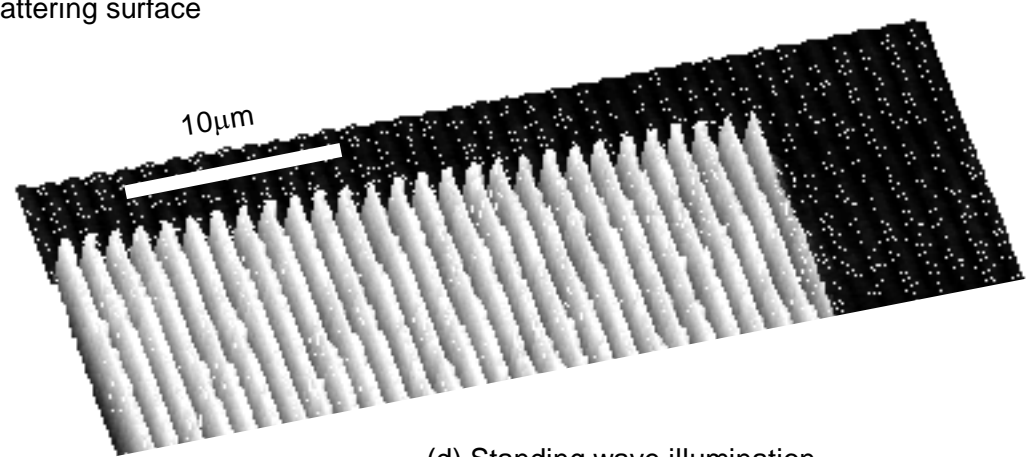

(d) Standing wave illumination

Fig. 15 Generation of standing wave illumination

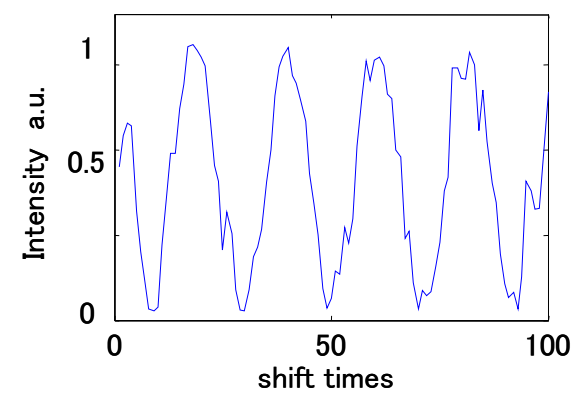

Fig. 16 Modulation of scattered light from a particle

\subsection{Super-resolution experiment using line and space sample}

For experimental verification of resolution enhancement using the proposed super-resolution method, we performed the super-resolution experiment using a 
500-nm line and space sample shown in Fig. 17, that was observed with an optical microscope $(\mathrm{NA}=0.95$, Rayleigh limit $=300 \mathrm{~nm})$. The experimental conditions are shown in Table 3. It is expected that the proposed super-resolution method makes it possible to observe a structure with higher resolution beyond the Rayleigh limit, depending on the NA of an objective lens. And as the verification of the fundamental principle of the method, we used an objective lens with relatively low NA $(0.46$, Rayleigh limit $=647 \mathrm{~nm})$ in the experiment. In scattered light imaging (dark-field imaging), the scattered light (diffracted light) from line edges is mainly detected with the photo-detector, so the $500 \mathrm{~nm}$ spaced line edges are expected to be resolved with the super-resolution method. Fig. 18 shows the dark-field scattered light image of the sample with 0.95 NA imaging optics. The $500 \mathrm{~nm}$-spaced line edges were resolved because the Rayleigh limit in this case was $313 \mathrm{~nm}$. In contrast, the dark-field image of the sample with 0.46 NA imaging optics is shown in Fig 19. In this figure, the line edges were not resolved because the Rayleigh limit in this case was $647 \mathrm{~nm}$. The sample was then illuminated with the standing wave, so multiple images of modulated scattered light were obtained, for which intensity distributions are shown in Fig 20. The results of the super-resolution reconstruction using multiple images are shown in Fig. 21, allowing us to determine the periodic intensity distribution. Furthermore, the sample was tilted to find out whether the line and space were appropriately resolved. The results and the tilt of the sample are shown in Fig 22, in which (a) shows the 522-nm line and space (tilt = 17 deg) result, and (b) shows 777-nm line and space (tilt $=50 \mathrm{deg}$ ) result. The Fig 21 and the Fig. 22 show the periodic distributions of the super-resolution results were corresponding to edges of the line and space sample, and the 500nm-spaced line edges are clearly resolved beyond the Rayleigh limit (647 nm) by using the super-resolution method. 


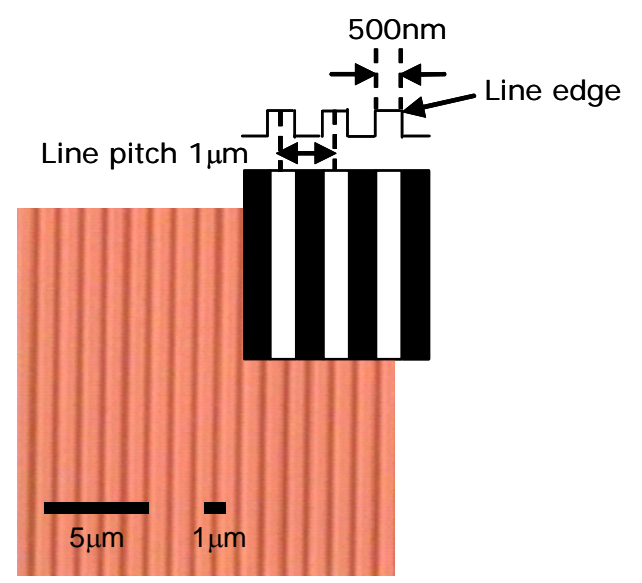

Fig. 17 Line and space sample

Table 3 Conditions of the super-resolution experiment

\begin{tabular}{c|c}
\hline \hline Pitch of standing wave illumination: $T$ & $1750 \mathrm{~nm}$ \\
$N A$ of objective: $N A$ & 0.46 \\
Magnification of imaging optics: $m$ & 38 \\
Standing wave shift step size: $S_{\text {shift }}$ & $80 \mathrm{~nm}$ \\
Illumination shift times: $t_{\text {shift }}$ & 24 \\
Iteration times: $i$ & 10 \\
\hline
\end{tabular}
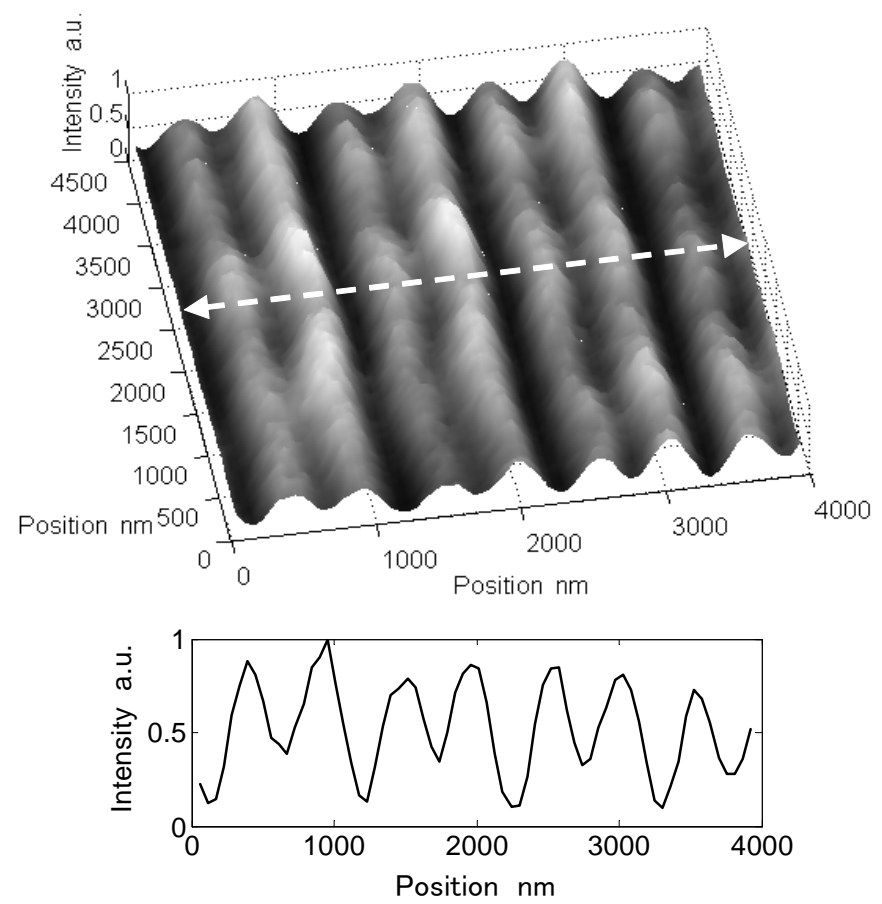

Fig. 18 Dark-field scattered light image of sample: $N A=0.95$ 


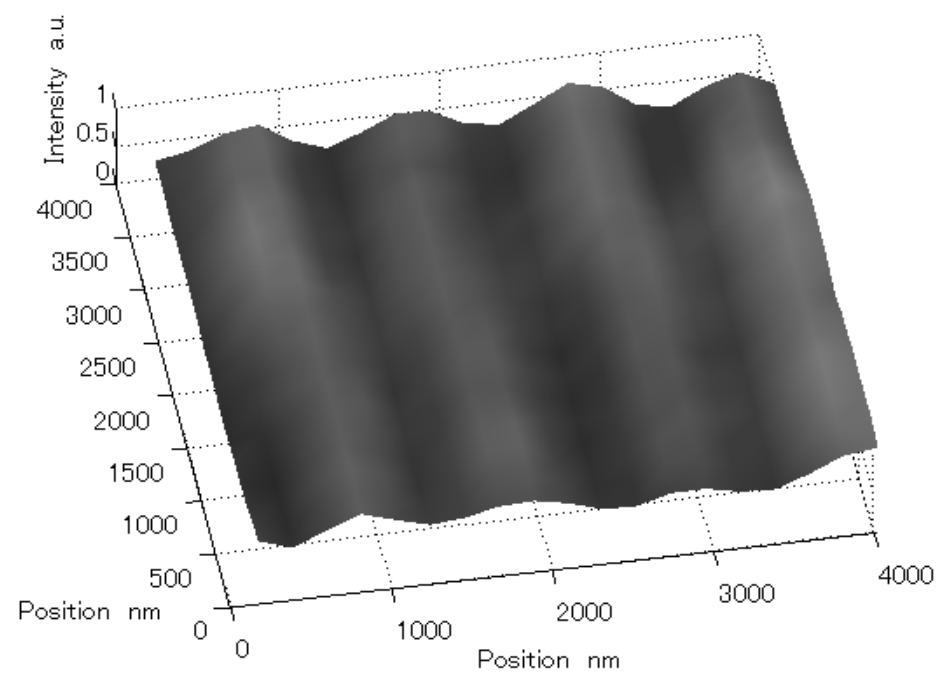

Fig. 19 Dark-field scattered light image of sample: $N A=0.46$

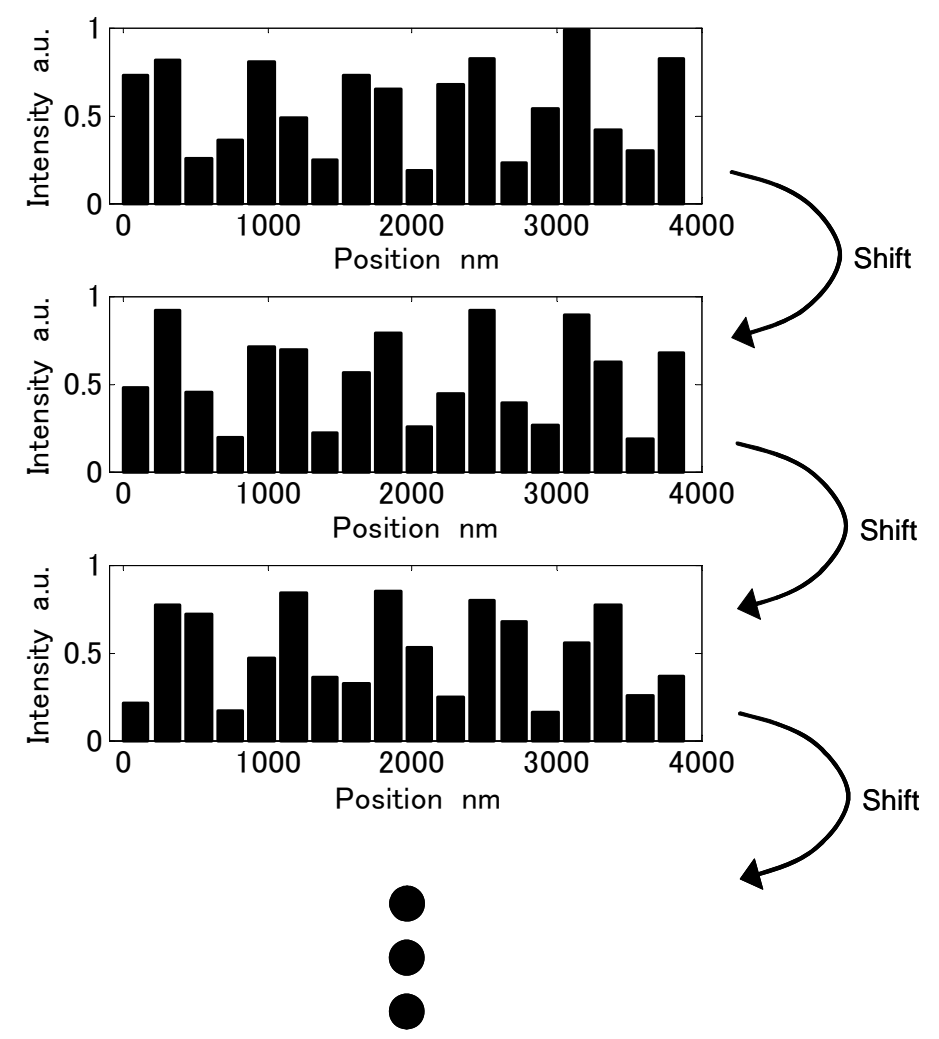

Fig. 20 Multiple images with standing wave illumination shift 

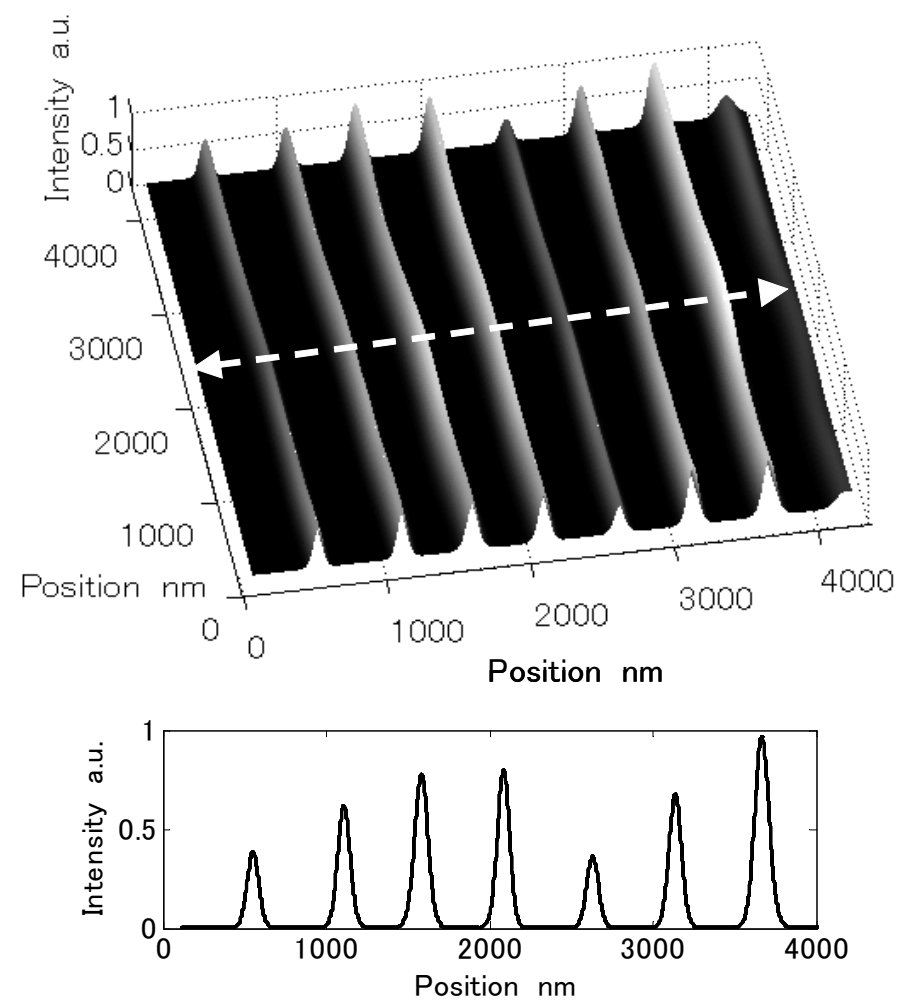

Fig. 21 Super-resolution image of periodic line edges

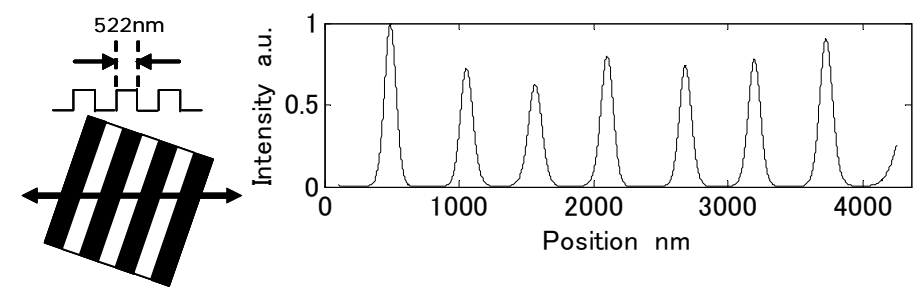

Tilt $17 \mathrm{deg}$.

(a) Super-resolution image with $17 \mathrm{deg}$ tilt
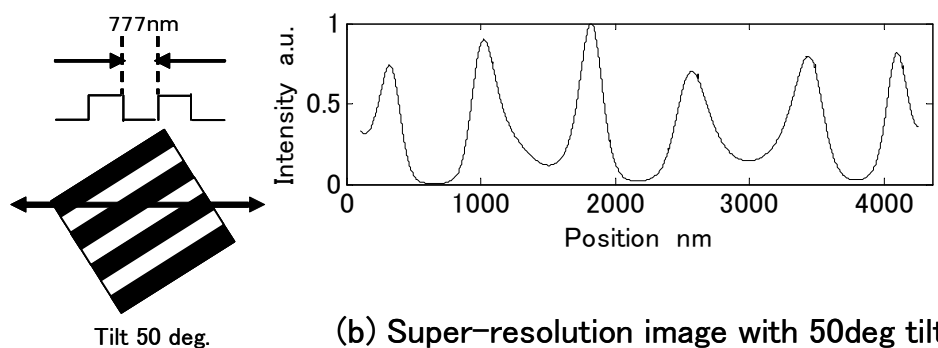

(b) Super-resolution image with 50deg tilt

Fig. 22 Super-resolution distribution of tilted line edges

\subsection{Super-resolution experiment for defect detection on the line and space}

The proposed method has been developed to be applied to semiconductor defect inspection, so a super-resolution defect detection experiment was performed. 
The conditions of this experiment were shown in Table 4. The Fig. 23 shows a SEM image of the sample including defect which is placed between 250-nm spaced line edges. The proposed super-resolution method was applied to resolve this sample and to detect the defect with 313-nm Rayleigh-limited imaging optics. The super-resolution image with the iterative reconstruction of multiple images with standing wave illumination shift is shown in Fig. 24. In this figure, it was found that defect placed between the $250-\mathrm{nm}$ spaced line edges was around the central position of the super-resolution image. It was therefore confirmed that the approximately $250 \mathrm{~nm}$-sized defect on the sample was detected with the proposed method with higher resolution than the Rayleigh limit of imaging optics (313 nm).

Table 4 Conditions of the super-resolution experiment for defect detection

\begin{tabular}{c|c}
\hline \hline Pitch of standing wave illumination: $T$ & $490 \mathrm{~nm}$ \\
NA of objective: $N A$ & 0.95 \\
Magnification of imaging optics: $m$ & 165 \\
Standing wave shift step size: $S_{\text {shift }}$ & $20 \mathrm{~nm}$ \\
Illumination shift times: $t_{\text {shift }}$ & 24 \\
Iteration times: $i$ & 20 \\
\hline
\end{tabular}

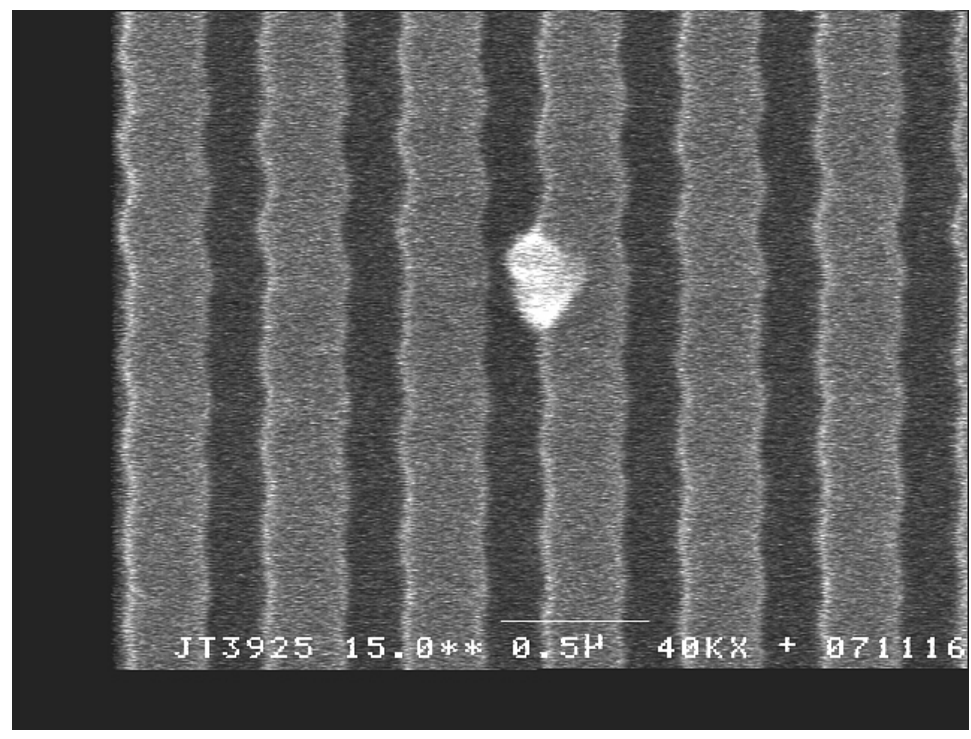

Fig. 23 SEM image of 250-nm spaced line and space sample with defect 


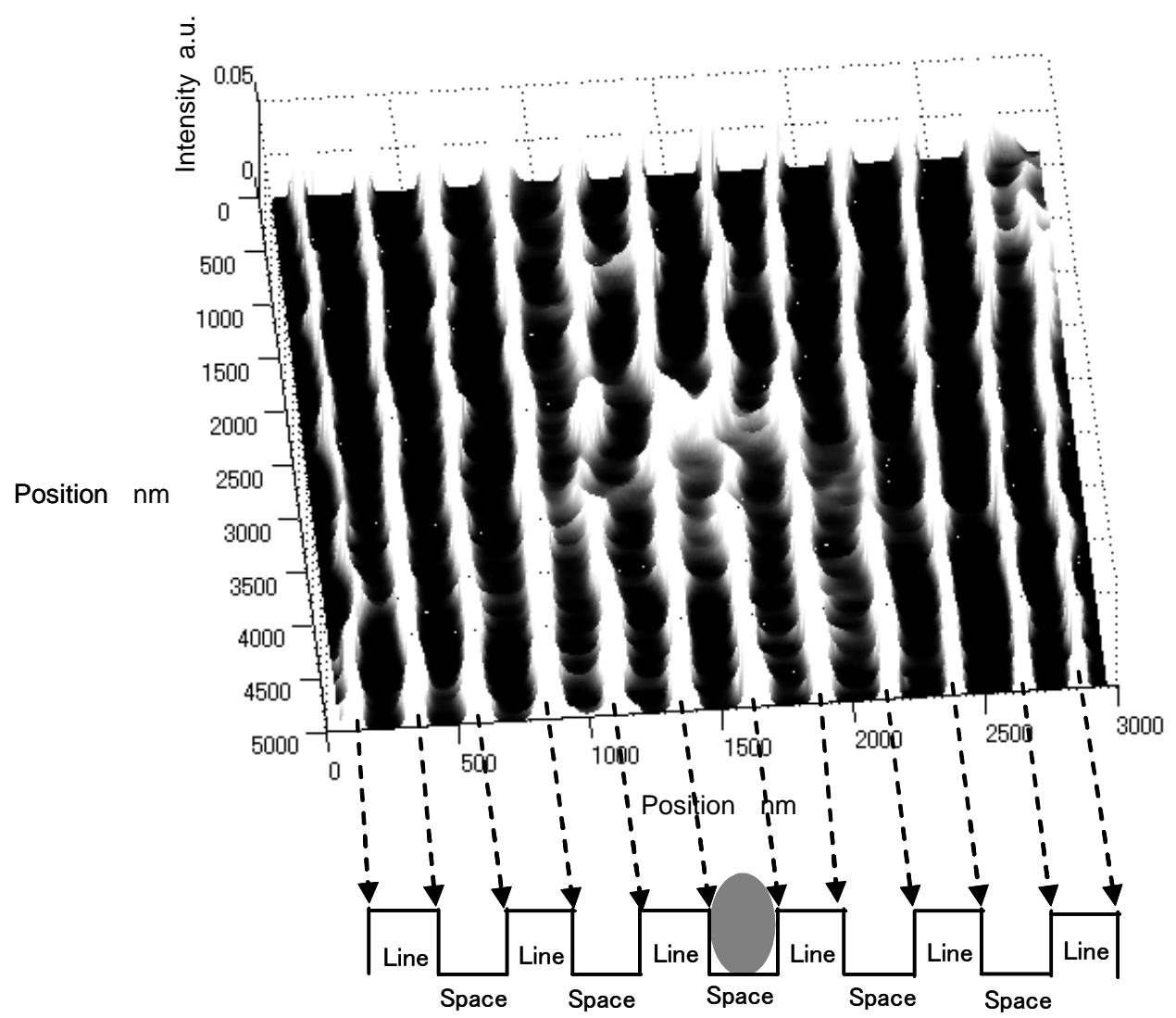

Fig. 24 Super-resolution image of 250-nm spaced line edges with defect

\section{Conclusions}

In order to verify the proposed super-resolution method, we carried out theoretical examinations including computer simulations to resolve 2-point objects with super-resolution under random noises, and we carried out super-resolution experiment to resolve the 500-nm line and space sample beyond the Rayleigh limit of an imaging optics (647nm), and also experiment to detect a defect placed between 250-nm spaced line edges. As a result, the super-resolution properties were verified theoretically and the 500nm-spaced periodic edge patterns were clearly resolved beyond the Rayleigh limit (647nm), and the 250nm-sized defect between line edges was detected. It is therefore suggested that the proposed superresolution method can be used for optical defect inspection of the next generation of semiconductor wafer. 


\section{References}

[1] International Technology Roadmap for Semiconductors, Metrology 2006 update, Metrology, Semiconductor Industry Association

[2] S.Kohyama: SoC Solutions and Technologies for Digital Hypermedia Platform, IEEE International Electron Devices Meeting, Technical Digest (1999) pp.8-13

[3] Kenji Watanabe, Shunji Maeda, Tomohiro Funakoshi and Yoko Miyazaki, Hitachi Review Vol. 54, No. 1, pp22-26, 2005

[4] Volker Westphal and Stefan W. Hell, PHYSICAL REVIEW LETTERS, No.143903, 2005

[5] S. Usuki, H. Nishioka, S. Takahashi and K. Takamasu: Super-resolution optical inspection for semiconductor defects using standing wave shift, Proc. of SPIE ISOT2005, (2005) 60490C-1.

[6] Jan T. Frohn, Helmut F. Knapp, and Andreas Stemmer: True optical resolution beyond the Rayleigh limit achieved by standing wave illumination, PNAS, 13, 97(2000), pp7232-7236

[7] H. Nishioka et al: A Super-Resolution Microscopy with Standing Evanescent Light and Image Reconstruction Method, Proc. of IMEKO World Congress., 12, TC2 (2006).

[8] S. Usuki, H. Nishioka, S. Takahashi, K. Takamasu: Experimental Verification for Superresolution Optical Inspection for Semiconductor Defect by using Standing Wave Illumination Shift, Proceedings of ISMTII 2007, (2007), pp387-390. 